\title{
Single-drug vinorelbine as a salvage re-induction regimen for 4 consecutive pediatric patients with relapsed anaplastic large-cell lymphoma in a single children's institution
}

\author{
Qing Yuan ${ }^{1}$, Qiao $\mathrm{He}^{1}$, Qiang $\mathrm{Mi}^{1}$, Min-Zhi Yin ${ }^{1}$, Yali $\operatorname{Han}^{1}$, and Yijin Gao ${ }^{1}$ \\ ${ }^{1}$ Shanghai Childrens Medical Center Affiliated to Shanghai Jiaotong University School of \\ Medicine
}

May 18, 2020

\begin{abstract}
Approximately $20-40 \%$ of pediatric patients with anaplastic large-cell lymphoma (ALCL) develop recurrent disease. Here, we report a pilot experience using single-drug weekly vinorelbine $(25 \mathrm{mg} / \mathrm{m} 2 /$ wk $)$ as a salvage re-induction regimen in 4 consecutive pediatric patients with relapsed ALCL. All 4 patients achieved complete remission after 2 cycles of weekly vinorelbine and were disease-free alive at last evaluation. The main toxicity was hematologic. Only one week of vinorelbine administration was withheld for a grade 4 neutropenia in one patient. The results suggest that weekly vinorelbine has good efficacy and safety in the treatment of relapsed pediatric ALCL.
\end{abstract}

\section{Introduction}

Anaplastic large cell lymphoma (ALCL) accounts for 10-15\% of all childhood non-Hodgkin lymphoma (NHL) cases [1]. In most series, 20-40\% of the patients develop recurrent disease. One of the unique features of ALCL is its sensitivity to chemotherapy after recurrence, leading to a survival rate of more than $90 \%$ in the ALCL99 study [2,3]. Although there is no standard approach for the treatment of recurrent ALCL, a regimen of single-drug weekly vinblastine has been shown to be highly effective for pediatric patients with relapsed ALCL, yielding an $83 \%$ complete remission (CR) rate with 9 of 25 of these patients experiencing continuous remission after a median follow-up of 7 years from the end of treatment [4]. However, vinblastine would not be advised as a treatment option for relapsed ALCL in mainland China due to its inaccessibility (drug shortage).

Vinorelbine is a semi-synthetic vinca alkaloid approved by the U.S. Food and Drug Administration in 1994. Vinca alkaloids are the earliest developed microtubule-targeting agents and have high anti-tumor potential as they interfere with the continuous mitotic division of cancer cells and thereby disrupt their abnormal growth [5]. Vinorelbine has a broad spectrum of anticancer activity, especially in the treatment of advanced/metastatic non-small cell lung cancer and breast cancer, either as a single agent or in combination with other drugs [5]. It is also worth noting that vinorelbine has demonstrated marked single-agent clinical activity $(50 \%)$ in patients with heavily pre-treated relapsed Hodgkin's lymphoma (HL) and ranks as one of the active agents in the management of HL [6]. This evidence suggests that this agent is effective in CD30+/ALK+ disease. In the pediatric setting, the maximum tolerated dose is $30 \mathrm{mg} / \mathrm{m}^{2} /$ week [7]. The current study reports a pilot experience using single-drug vinorelbine as a salvage re-induction regimen in pediatric patients with relapsed ALCL.

\section{Methods}


All patients with relapsed ALCL included in the study were initially treated in our hospital in 2016-2018. Histology confirmation was required for the diagnosis of relapsed ALCL in all patients. Detailed data on the first- and second-line treatments and the outcomes of these patients were collected from their medical files.

A cycle of therapy was defined as a 4-week interval during which vinorelbine was given as a weekly intravenous injection of $25 \mathrm{mg} / \mathrm{m}^{2}$ for 3 consecutive weeks (days 1,8 , and 15 ) followed by a 1 -week rest. Steroids were combined with vinorelbine during the first 4 to 6 weeks of the treatment. At least 2 cycles of weekly vinorelbine regimen were administered unless there were signs of disease progression. After 2 cycles, a formal assessment by computed tomography (CT) or magnetic resonance imaging (MRI) scans was performed. Fluorodeoxyglucose positron emission tomography scan is recommended but not mandatory. The treatment after 2 cycles of single-drug vinorelbine therapy was at the discretion of the physician. Any further single-drug vinorelbine therapy was only to be administered if $\mathrm{CR}$ was achieved after the initial two cycles.

Complete remission was defined as disappearance of all evidence of disease. Unconfirmed complete response was defined as a residual lymph node mass $>1.5 \mathrm{~cm}$ on CT scans, or MRI evaluations that regressed by $>75 \%$ in the sum of the products of the greatest perpendicular diameters (SPD) of the lesions, or any residual lesions in organs that decreased by $>75 \%$ with a negative PET scan. Progressive disease was defined as an increase of $>25 \%$ in SPD or the development of a new lesion.

Toxicity was graded according to the Common Terminology Criteria for Adverse Events, version 3.0. Hematological and hepatic toxicity rates were estimated. Dose reductions of vinorelbine were not permitted in this protocol. Vinorelbine was withheld at either the second or third dose (days 8 or 15, respectively) of each vinorelbine cycle for an absolute neutrophil count less than $500 / \mathrm{mm}^{3}$ or platelets $<50,000 / \mathrm{mm}^{3}$. If a dose was withheld, it was not repeated.

\section{Results}

Four consecutive patients aged between 4 and 13 years at initial diagnosis were treated. The disease relapse was confirmed through biopsy in all 4 patients. All the patients were initially treated according to CCCGBNHL-2010 (a 6-month chemotherapy regimen combining vincristine, doxorubicin, cyclophosphamide, prednisone, high-dose methotrexate, etoposide, and triple intrathecal therapy) [8].

All 4 patients achieved a CR after 2 cycles of weekly vinorelbine. Patients 1 and 2 underwent autologous hematopoietic stem-cell transplantation (HSCT) afterwards, and both had a second relapse, 18 months and 24 months after the first one, respectively. Fortunately, those 2 patients achieved their CR3 after receiving 3 cycles of multiagent chemotherapy combined with crizotinib. Both refused further allogeneic HSCT but continued on crizotinib and remained in CR3 at last evaluation. Patient 3 underwent allogeneic HSCT afterwards and remained in his CR2. Patient characteristics, treatments, and outcomes are shown in Table 1.

The main toxicity related to weekly vinorelbine was hematologic. In total, 4 patients received 24 cycles of weekly vinorelbine. Table 2 summarizes the grade 3 and 4 toxic effects. Only 1 week of vinorelbine administration was withheld, for a grade 4 neutropenia in Patient 1.

\section{Discussion}

The major challenge in treating pediatric ALCL is its high rate of relapse. Event-free survival rates have barely changed since the NHL-BFM first tested its B-cell NHL protocol on ALCL patients in the 1980s. Vinblastine has unusual efficacy as a single agent in relapsed ALCL when given for prolonged durations $[2,3,9]$. The experience with single-agent vinblastine in relapse therapy suggested it could be a potential drug in frontline therapy for pediatric ALCL. However, vinblastine inaccessibility poses a particular problem in mainland China and in the treatment of conditions such as relapsed ALCL [10].

This is the first report of single-drug vinorelbine in pediatric relapsed ALCL. All 4 patients achieved a CR. These results are comparable to the data from previous single-drug vinblastine studies for relapsed ALCL. Vinorelbine belongs to the second-generation vinca alkaloid compounds, which are more potent and less 
neurotoxic than vinblastine and vincristine. In addition, the availability of an oral formulation may be of particular interest in the treatment of ALCL. Maintenance therapy was shown to be highly efficient in relapsed ALCL and may produce durable remission [4].

Of interest, all 4 included patients received very high doses of vincristine in their initial treatment, suggesting an absence of cross-resistance between vinorelbine and other vinca alkaloid compounds. Indeed, the antiproliferative cascade triggered by vinca alkaloids at the molecular level is still unclear. About $90 \%$ of pediatric ALCL patients are positive for ALK fusion proteins, most of them caused by the $\mathrm{t}(2 ; 5)(\mathrm{p} 23 ; \mathrm{q} 35)$ translocation. These translocations induce constitutive phosphorylation of ALK that activates multiple pathways such JAK/ATAT3, AKT/P13K, and RAS/ERK, leading to growth factor-independent cell proliferation and inhibition of apoptosis. The ERK pathway was found to be relevant to clinical response in predicting the effectiveness of vinorelbine in patients with non-small cell lung cancers [11].

In our series, the toxicity of vinorelbine has no impact on treatment schedule. Our findings suggest that vinorelbine is a potential therapeutic option for pediatric patients with recurrent ALCL and that the feasibility of delivering this regimen is satisfactory. The efficacy and safety of this single-drug regimen deserve to be evaluated on a large scale. We are now designing a clinical trial based on single-drug vinorelbine as salvage treatment for recurrent ALCL, which should be of interest in regard to pediatric patients with recurrent ALCL in China.

Disclosures: The authors have no conflicts of interest or funding to disclose.

\section{References}

1. Burkhardt B, Zimmermann M, Oschiles I et al. The impact of age and gender on biology, clinical features and treatment outcomes of non-Hodgkin lymphoma in childhood and adolescence. Br J Haematol 2005; 131: $39-49$.

2. Brugières L, Le Deley MC, Rosolen A et al. Impact of the methotrexate administration dose on the need for intrathecal treatment in children and adolescents with anaplastic large-cell lymphoma: Results of a randomized trial of the EICNHL Group. J Clin Oncol 2009; 27: 897-903.

3. Le Deley MC, Rosolen A, Williams DM et al. Vinblastine in children and adolescents with high-risk anaplastic large-cell lymphoma: Results of the randomized ALCL99-vinblastine trail. J Clin Oncol 2010; 28: 3987-3993.

4. Brugières L, Pacquement H, Le Deley MC et al. Single-drug vinblastine as salvage treatment for refractory or relapsed anaplastic large-cell lymphoma: a report from the French Society of Pediatric Oncology. J Clin Oncol 2009; 27: 5056-5061.

5 Emanuela M, Giuseppe C, Sonia C et al. Vinca alkaloids and analogues as anti-cancer agents: looking back, peering ahead. Bioorg Med Chem Lett 2018; 28: 2816-2826.

6. Devizzi L, Santoro A, Bonfante V et al. Vinorelbine: An active agent for the management of patients with heavily pretreated Hodgkin disease. Ann Oncol 1994; 5: 817-820.

7. Kuttesch JF, Krailo MD, Madden T et al. Phase II evaluation of intravenous vinorelbine (Navelbine) in recurrent or refractory pediatric malignancies. A Children's oncology group study. Pediatr Blood Cancer 2009; 53: 590-593.

8. Subspecialty Group of Hematology, the Society of Pediatrics, Chinese Medical Association, Pediatric Oncology Committee, Chinese Anticancer Association, Editorial Board of Chinese Journal of Pediatrics. Recommendation for pediatric non-Hodgkin's lymphoma [in Chinese]. Zhonghua Er Ke Za Zhi 2011; 49: 186-192.

9. Brugières L, Quartier P, Le Deley MC et al. Relapses of childhood anaplastic large-cell lymphoma: Treatment results in a series of 41 children-a report from the French Society of Pediatric Oncology. Ann Oncol 2009; 11: 53-58. 
10. Lymphoma Study Group, Subspecialty Group of Hematology, the Society of Pediatrics, Chinese Medical Association; Lymphoma Study Group, Committee of Pediatrics, Chinese Anti-Cancer Association. Diagnosis and Treatment of Anaplastic Large-Cell Lymphoma in Children and Adolescents: A Retrospective Multicenter Survey Study [in Chinese]. Zhonghua Er Ke Za Zhi 2017; 55: 194-199.

11. Shi Y, Chen L, Li J et al. Prognostic and predictive value of pERK1/2 and pAkt-1 expression in non-small cell lung cancer patients treated with adjuvant chemotherapy. Tumor Biol 2011; 32: 381-390.

\section{Hosted file}

table 1 20200516. docx available at https://authorea.com/users/323048/articles/451970-singledrug-vinorelbine-as-a-salvage-re-induction-regimen-for-4-consecutive-pediatric-patientswith-relapsed-anaplastic-large-cell-lymphoma-in-a-single-children-s-institution

\section{Hosted file}

table 2 20200516.docx available at https://authorea.com/users/323048/articles/451970-singledrug-vinorelbine-as-a-salvage-re-induction-regimen-for-4-consecutive-pediatric-patientswith-relapsed-anaplastic-large-cell-lymphoma-in-a-single-children-s-institution 